\title{
Three Point Mutations in the Factor IX Genes of Five Hemophilia B Patients Identification Strategy Using Localization by Altered Epitopes in Their Hemophilic Proteins
}

\author{
Shi-Han Chen, Arthur R. Thompson, Min Zhang, and C. Ronald Scott \\ Departments of Pediatrics and Medicine, University of Washington and the Puget Sound Blood Center, Seattle, Washington 98195
}

\begin{abstract}
In five patients with hemophilia B and detectable Factor IX antigen, altered reactivity to a specific polyclonal antibody fraction or monoclonal anti-Factor IX antibodies was noted. Amplification of selected portions of their Factor IX genes by polymerase chain reaction allowed rapid identification of a single base transition in each of the five families tested. In a patient with severe hemophilia and an altered calcium binding domain, a $\mathbf{G}$ to $A$ transition in exon II changed the codon for Glu-27 to Lys (Factor IX Seattle 3). Patients from two families with mild hemophilia with decreased reactivity to a MAb that binds to a site within the sequence coded for by exon IV had a $G$ to A transition changing the codon for Gly-60 to Ser (Factor $\left.\mathrm{IX}_{\text {Durham }}\right)$. Two unrelated patients with moderately severe hemophilia lacked reactivity to another murine monoclonal antiFactor IX which binds to an epitope in the amino-terminal half of the heavy chain of Factor IXa. In these patients, exon VIII contained a $G$ to A transition changing Arg-248 to Gln (Factor IX $\mathbf{X}_{\text {Seattle 4). }}$
\end{abstract}

\section{Introduction}

Hemophilia B is an X-linked bleeding disorder caused by deficiency of Factor IX clotting activity in patients' bloods. Genetic heterogeneity is reflected by varied clinical severity and different levels of Factor IX activities and antigen found in plasmas of affected members from different families (1). The known molecular defects of Factor IX in patients with hemophilia B are likewise heterogeneous, including gene deletions, an insertion, and point mutations (2). Because of the lack of a selective advantage of hemophilia, it can be assumed that each family has a "private" defect (3). Occasionally, patients from different families will have the same defect (e.g., references 4-6), suggesting the presence of mutation hot spots.

Recently, additional point mutations are being defined by using the polymerase chain reaction and sequencing the amplified DNA fragments (e.g., references 7-9). In the current study, amplification and DNA sequencing were performed on selected regions of the Factor IX gene. These regions were identified because of decreased binding of the respective hemophilic Factor IX proteins to antibodies known to react with

Address correspondence to Dr. Shi-Han Chen, Division of Pediatric Genetics, University of Washington, Mail-stop RD-20, Seattle, WA 98195.

Received for publication 4 January 1989 and in revised form 1 March 1989.

J. Clin. Invest.

(c) The American Society for Clinical Investigation, Inc.

0021-9738/89/07/0113/06 \$2.00

Volume 84, July 1989, 113-118 epitopes in distinct sequence regions of the Factor IX protein. Each patient had detectable hemophilic Factor IX proteins that were characterized immunochemically. Three distinct point mutations in five different families were identified.

\section{Methods}

Factor IX assays. Factor IX clotting activities were determined in the one-stage clotting assay using deficient substrate plasmas as described (1). Factor IX antigen levels were from immunoradiometric assays using fractions of polyclonal rabbit anti-Factor IX sera (10). For some assays, MAbs were used as the solid phase (adsorbed) reagent with labeled polyclonal rabbit anti-Factor IX fractions as the second antibody. MAbs included C10D (11), 2D5 provided by Howard Reisner, Chapel Hill, NC (12), and A-1 and A-7 from Kenneth Smith, Albuquerque, NM (13).

Purification and electrophoresis of hemophilic Factor IXs. Heparinized plasma samples $(5-15 \mathrm{ml})$ were immunoaffinity purified over a column ( $1 \mathrm{ml}$ bed volume for $1-5 \mathrm{ml}$ plasma) of $\mathrm{CH}$-Sepharose to which $1 \mathrm{mg} / \mathrm{ml}$ rabbit calcium-dependent polyclonal antibody had been attached. Columns were equilibrated in $5 \mathrm{mM} \mathrm{CaCl}_{2}$ and the partially purified Factor IX eluted in $10 \mathrm{mM}$ EDTA. For the propositus in family B-2, a 500-ml sample of plasma was available and his

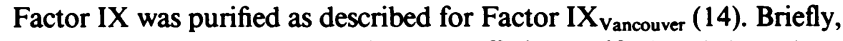
the barium citrate eluate was immunoaffinity purified and then chromatographed on a mono-Q column. This material was then radioiodinated as described (1). PAGE in SDS was performed as described (15) for partially purified patient vs. normal Factor IXs which were immunoblotted, or by transfer to nitrocellulose and direct radioautography for ${ }^{125}$ I-labeled Factor IXs.

DNA samples, purification, and polymerase chain reaction $(P C R) .^{\prime}$ DNA was extracted from leukocytes prepared from 10-20 ml whole blood anticoagulated with either heparin or EDTA and genomic DNA isolated as described (16). The PCR was performed according to a modified method described by Saiki et al. (17) with an automatic DNA thermal cycler (Perkin Elmer-Cetus, Norwalk, CT). The conditions were set at $94^{\circ} \mathrm{C}$ for $1 \mathrm{~min}$ for denaturation, followed by $1.5 \mathrm{~min}$ at $50^{\circ} \mathrm{C}$ for annealing, and $2 \mathrm{~min}$ at $70^{\circ} \mathrm{C}$ for extending. A total of 30 cycles were performed. Three sets of primers (20-25 mers) were used to amplify exon II, exon IV, and the $5^{\prime}$ half of exon VIII and their exon/ intron junctions. The locations and directions of the primers are shown in Fig. 1. The lengths of the amplified fragments are $338 \mathrm{bp}$ for exon II, 273 bp for exon IV, and 363 bp for exon VIII.

DNA sequencing. The amplified DNA fragments were purified and subjected to direct nucleotide sequencing by the dideoxy chain termination method of Sanger et al. (18). The sequence kit and procedure were obtained from United States Biochemical Corp., Cleveland, $\mathrm{OH}$. The primers used for amplification served as primers for sequencing. After completing the sequencing reaction the samples were applied to a $6 \%$ polyacrylamide/urea gel and run at $65 \mathrm{~W}$ for $2-4 \mathrm{~h}$. The gel was fixed in 5\% methanol, $5 \%$ acetic acid, dried, and exposed to $x$-ray film (XAR5; Eastman Kodak, Co., Rochester, NY) for 1-3 d. Under these conditions $150-200$ bp of sequence could be read from the ladder. All five patients' DNA were amplified and sequenced both directions through the three fragments to serve as internal controls.

1. Abbreviations used in this paper: $\mathrm{PCR}$, polymerase chain reaction. 


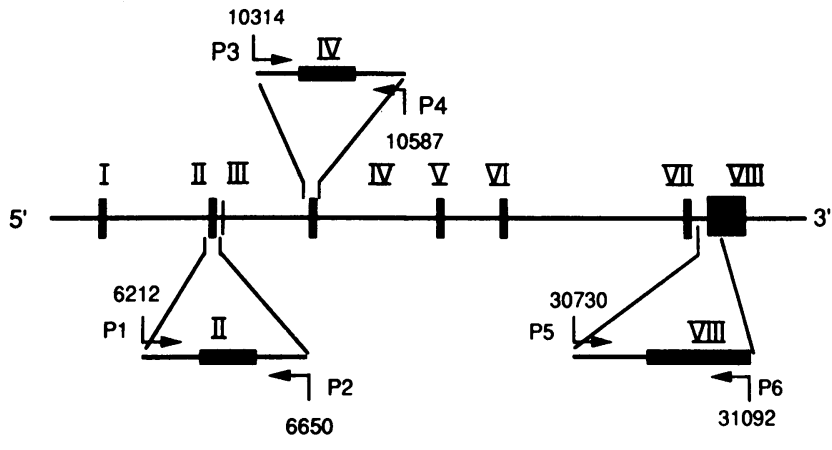

Figure 1. Factor IX gene organization and strategy of PCR amplifcation and sequencing of patients' DNA. The schematic diagram for the gene $(\sim 33.5 \mathrm{~kb})$ with its eight exons (solid bars) is shown. The locations of the primers are in the enlarged portions of the maps above and below the gene. Primer sequences and directions (arrows) are given and numbered according to the nucleotide sequence of Yoshitake et al. (22), including P1, ${ }^{\prime}$ GAAATTGGCTTTCAGATTAT (6212-6231); P2, 5'ACGGTAATATCTAAAGTGTT (6650-6631); P3, ${ }^{5}$ CCAATGAGTATCTACAGGGG (10314-10333); P4, ${ }^{5 \prime}$ TACACCAATATTGCATTTTC (10587-10568); P5, 5'GCCAATTAGGTCAGTGGTCC (30730-30749); and P6, ' ${ }^{\prime}$ TGAAGAACTAAAGCTGATCT (31092-31073). Of these, P2, P4, and P6 represent reverse or antisense sequences.

Patients. Affected members from 115 families with hemophilia B were screened for Factor IX antigen levels by several immunoassays. Samples were provided with informed consent as approved by the Human Subject Review Committee of the University of Washington. One-third were clinically severe and of these, six had $<0.1 \mathrm{U} / \mathrm{dl}$ baseline Factor IX antigen. In three specific immunoassays, patients from six other families had decreased Factor IX antigen levels relative to their total Factor IX antigen. At the times of sample collection these patients had not been treated for at least $4 \mathrm{wk}$. Immunologic results in the six families are referred to as A, B-1 to -3, C-1 and -2, and DNA was available in all but B-3. All but the elder member of B-2 had a positive history of hemophilia in earlier generations.

Patient A was 36 yr old with a lifelong history of joint and muscle bleeds, both spontaneously and after trauma. His clotting activity was $<1 \mathrm{U} / \mathrm{dl}$ with a mildly reduced antigen level. He had received replacement therapy since childhood and had advanced hemophilic arthropathy in several joints. The hemophilic gene could be traced to four generations of his family.

Patients from the families in group B all had mild hemophilia B, having required only occasional replacement therapy for bleeding induced by major trauma or surgery. Factor IX clotting activities ranged from 15 to $25 \mathrm{U} / \mathrm{dl}$ with total antigen levels from 25 to $55 \mathrm{U} / \mathrm{dl}$. In family B-1, plasmas from five affected members were tested (see Fig. 5 and Table III in reference 10). Their hemophilic gene was present for at least five generations. Family B-2 consisted of a 62 -yr-old man and his 6-yr-old grandson. Only occasional bleeding episodes were encountered throughout life and were managed with plasma. Patient B-3 was last seen at age 12 before moving. He had only required plasma once for prolonged oozing after tooth extractions.

The patients in group $\mathrm{C}$ had moderately severe hemophilia $\mathrm{B}$ with low Factor IX antigens and were previously reported as not having detectable reactivity to a murine monoclonal anti-Factor IX antibody that reacts with an epitope within the heavy chain of Factor IXa (11). Patient C-1 was 72 yr old and in need of hip surgery. When he was younger he had several bleeding episodes related to trauma but required little therapy over the past several years. By history, his maternal grandfather was affected. Patient C-2 was 37 yr old and had several deep muscle hematomas related to minor injuries that required prolonged treatment. His hemophilic gene was presented for at least three generations. There was no living ancestor available for testing in any of the five families who represented a potential origin of the mutation.

\section{Results}

Immunoassays on hemophilic Factor IX proteins. Table I presents the Factor IX levels as clotting activity, total antigen, and antigen by three immunoradiometric assays with more specific antibodies. Patients from the six different families tested were grouped according to reactivity in the specific assays where the epitopes primarily reflect the Gla-domain (A), the first epidermal growth factor-like region (B), and the amino-terminal half of the heavy chain of Factor IXa (C). It should be noted that patients from each of these families had decreased reactivity in a single, specific assay with comparable levels in other assays. None of the other 109 patients, nor a separate series of 35 normal Caucasian male donors, had $>15 \%$ difference in any of their specific vs. total clottable Factor IX antigen levels.

The patient in family A had mildly reduced total Factor IX antigen with levels from the calcium-dependent assay of only one-third of his total antigen level. In a separate immunoradiometric assay using as a solid-phase A-1 and as labeled antibody A-7 MAbs, where the latter is dependent on divalent metal ions $(13,15)$, his plasma level was $11 \mathrm{U} / \mathrm{dl}$. This value was the same as determined for the polyclonal, calcium-dependent system (Table I). The epitopes to which this antibody fraction binds, in the presence of calcium, are on the light chain of Factor IXa and reactivity is reduced in vitamin $\mathrm{K}$ disorders $(15,19)$. It was predicted that this patient's defect would involve the Gla domain, and, because of his severity and the relative importance of the more amino-terminal Gla residues of prothrombin, that the defect would be within exon II.

The B group of families were not known to be related and all had mild hemophilia with mildly reduced Factor IX anti-

Table I. Factor IX Levels in Six Patients' Plasmas

\begin{tabular}{|c|c|c|c|c|c|c|}
\hline \multirow[b]{2}{*}{$\begin{array}{c}\text { Patient } \\
\text { (family) }\end{array}$} & \multirow[b]{2}{*}{$\begin{array}{l}\text { Clinical } \\
\text { severity }\end{array}$} & \multicolumn{5}{|c|}{ Factor IX level } \\
\hline & & $\begin{array}{l}\text { Clotting } \\
\text { activity }\end{array}$ & $\begin{array}{l}\text { Total (p) } \\
\text { antigen }\end{array}$ & $\begin{array}{l}\mathrm{Ca}^{2+}(p) \\
\text { antigen }\end{array}$ & $\begin{array}{l}2 \mathrm{D} 5(\mathrm{~m}) \\
(50-84)\end{array}$ & $\begin{array}{l}\text { C10D (m) } \\
(181-310)\end{array}$ \\
\hline & & \multicolumn{5}{|c|}{$U / d l$} \\
\hline$A^{*}(1)$ & Severe & $<1$ & 30 & 10 & 29 & 29 \\
\hline B-1* (5) & Mild & 17 & 30 & 32 & 11 & 29 \\
\hline B-2* (2) & Mild & 11 & 19 & 20 & 7 & 19 \\
\hline B-3 (1) & Mild & 10 & 18 & 20 & 9 & 20 \\
\hline$C-1^{*}(1)$ & Moderate & 3 & 4 & 3 & 2 & $<0.1$ \\
\hline$C-2 *(1)$ & Moderate & 4 & 3 & 4 & 2 & $<0.1$ \\
\hline
\end{tabular}

Factor IX antigen levels were determined by immunoradiometric assays, including polyclonal $(p)$, nondivalent metal ion-dependent fraction for total antigen and $\mathrm{Ca}^{2+}$ antigen by divalent metal iondependent polyclonal anti-Factor IX fraction (15); 50-84, a murine monoclonal $(m)$ anti-IX, referred to as $2 \mathrm{D} 5$, with its epitope in the first epidermal growth factor-like region $(12,20)$; and 181-310, another murine monoclonal reacting with the amino-terminal half of the heavy chain of IXa $(11,12)$.

* DNA analysis reported in the current study. Numbers in parentheses are numbers of affected members immunoassayed; where samples from more than one affected individual were tested, results from a representative patient are presented. 
gen levels. Apparent antigen levels of one-fourth to one-half were found when 2D5 was the solid-phase antibody, compared with all other immunoradiometric assays (Table I). This ratio was present in all five affected members in family B-1 tested, the two affected members in B-2, and the one in B-3. This suggested that they all had the same or very similar defects. When the $0.1 \mathrm{M} \mathrm{NaCl}$ was omitted from incubations and washes in the immunoradiometric assays (lower ionic strength assays; not shown in table) there was insignificant change in the total Factor IX antigen with polyclonal antibodies, but in the solid-phase 2D5 immunoassay these patients showed an apparent twofold increase compared with controls. Washing after the sample incubation with $0.5 \mathrm{M} \mathrm{NaCl}$ instead of the usual $0.1 \mathrm{M} \mathrm{NaCl}$ reduced their apparent levels $20 \%$ further in the 2D5 assays. Thus, the reduced binding of patients' plasma Factor IXs to 2D5 was ionic strength dependent. Within family B-1, Factor IX clotting activities and antigen levels of affected members varied nearly twofold among different individuals. Where tested, these appeared to be constant in up to three separate samples from the same individual (see reference 10). Since 2D5 binds to an epitope within the sequences coded for by exon IV (12), it was predicted that patients from these three families would have an amino acid substitution within this amino-terminal epidermal growth factor-like region (20).

A different immunoradiometric assay using solid-phase A-1 antibody was used to test for the dimorphism at residue 148 (10). All patients in Table I except for the two in family B-2 were strongly reactive, indicating they had the Thr-148 allele. Hemophilia in the two patients from family B-2 was associated with the Ala-148 allele (e.g., markedly decreased binding to solid-phase A-1 MAb).

Although not known to be related, and from different ethnic and geographic lineage, the two patients, C-1 and C-2, had similar low levels of Factor IX activity and antigen and moder- ately severe bleeding tendencies. As previously reported (11), they lacked detectable binding to C10D, a MAb, which involves residues coded within the last 15 codons of exon VI, exon VII's 28 codons, and the first 77 codons of exon VIII (12). Over half of these exon VIII coding sequences are in variable regions of the heavy chain of Factor IXa (21). Therefore, it was predicted that exon VIII would most likely contain the substitution(s) in these patients.

Immunoelectrophoresis of hemophilic proteins. All patients' partially purified Factor IXs migrated the same as normal Factor IX on immunoblots after PAGE (7.5\%) in SDS. In addition to these results with partially purified Factor IXs, a purified sample from two patients in family B-1 and one from the affected member C-2 also showed normal mobility. After Factor XIa cleavage, the isolated, radiolabeled hemophilic Factor IX from patient B-2 was electrophoresed. Activation cleavages occurred to a comparable extent as with normal Factor IX and migration of both heavy and light chains was indistinguishable from the normal control.

Single point mutations and derived amino acid substitution. From the amplified patient DNA fragments, single point mutations were identified in patients of all five families tested. For the patient in family A, a G to A mutation (see Fig. 2 a) was found at nucleotide 6,454 in exon II. This changed the codon for Glu-27 to Lys, at a residue that is normally gamma carboxylated. For two patients in family B-1 and the propositus in B-2, G to A transitions (Fig. $2 b$ ) were found at nucleotide sequence 10,430 in exon IV. This will change Gly-60 of the normal Factor IX sequence to Ser in these patients. For families C-1 and C-2, a G to A transition was found at nucleotide 30,864 (Fig. 2 c). This mutation changes Arg-248 to Gln in the patients' Factor IXs. Fig. 2 also summarizes the three point mutations and their derived amino acid changes in the families. The three amplified sequences $(\sim 1,100$ bp sequenced) in

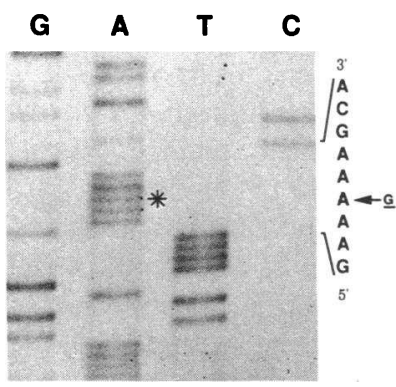

a

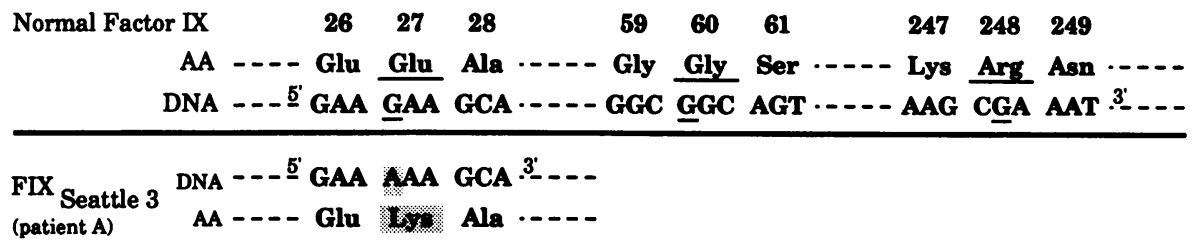

FIX Durham
(patients B-1 and B-2)

FIX Seattle 4

(patients C-1 and C-2)

$$
\text { DNA - - 5' AAG CAA AAT } 3 \text { - }
$$$$
\text { AA.-.- Lys alin Asn...- }
$$

Figure 2. Partial sequences of three point (G to A) mutations. (Top) Sequence gels with the altered and adjacent codons. $a, b$, and $c$ reflect hemophilic defects within exons II, IV, and VIII, respectively. Sequences are indicated on their right. The normal base is underlined (not shown in gels) and the mutant base is shaded. (Bottom) Partial nucleotide sequences are shown with derived amino acids of the normal and mutant and their adjacent codons. The substituted base and amino acid are underlined in the normal sequences and shaded in the hemophilic patients' sequences. Codon and amino acid residue numbers are from the amino terminus of the mature protein. $A A$, amino acid; $F I X$, Factor IX. 
patients with each of these three defects were otherwise as previously reported for normal Factor IX (22).

\section{Discussion}

A group of unrelated hemophilia B families was studied with affected members showing altered immunoreactivity to one of three specific antibody preparations. A strategy was devised to localize their defects, predicting that point mutations would be found within or near the regions of Factor IX that bind these antibodies in exons II, IV, or VIII among the five families tested. After PCR amplification and direct sequencing of the selected fragments of the gene, a single base transition in the suspected exon was found in each case.

In patient $A$ the defect was associated with severe hemophilia and altered calcium-binding properties. The Glu-27 to Lys mutation is designated Factor IX $\mathrm{X}_{\text {Seattle 3. }}$. Two patients with nucleotide changes in this region were recently reported by Koeberl et al. (23). In one, Arg-29 was Gln in a patient with 30 $\mathrm{U} / \mathrm{dl}$ Factor IX clotting activity (mild), and in a second, Glu-33 was substituted for Asp, producing a moderately severe bleeding tendency. In vitamin $\mathrm{K}$-dependent proteins, the homologue of Glu-27 is invariant and becomes a Gla residue; a Glu-33 homologue is not present in Factor VII or protein C, but is also a Gla in the other vitamin $\mathrm{K}$-dependent proteins. Characterization of abnormal prothrombin fractions from a patient with a congenital vitamin K-deficient state (24) suggests that fractions without Gla-27 (residue 26 in human prothrombin) are inactive. In contrast, the residue homologous to Gla-33 did not appear responsible for either of two high-affinity calcium-binding sites necessary for divalent metal ion-dependent conformation or activity. Assuming similar conformations of the Gla domains of Factor IX and prothrombin, patient A not only lacks what may be an essential Gla residue, but has a substitution leading to a strong positive charge at that position which might further disrupt calcium binding.

Defects at the -4 and -1 amino acid positions are also coded within the second exon and result in severe hemophilia. Each has an extended 17 amino acid reflecting the uncleaved proleader sequence that appears to lead to an abnormal conformation of the Gla domain. Samples of partially purified Factor IX $\mathrm{IXxord} 3_{3}(4)$ and Factor IX $\mathrm{X}_{\text {Cambridge }}$ (25) plasma (provided by George Brownlee, Oxford, UK, and Bruce Furie, Boston, MA, respectively) had 80 and $60 \%$ as much apparent Factor IX antigen in calcium-dependent vs. total assays. When the monoclonal immunoradiometric assay using antibody A-7 was substituted, both Factor IX Oxford $3_{3}$ and Cambridge had apparent levels that were $30 \%$ higher than their total Factor IX antigen in contrast to the lower levels in patient $A$.

The patients in group B and two others from North Carolina were found to have low reactivity to a MAb known to bind to the first epidermal growth factor-like region of Factor IX (20). The derived substitution of a Gly to Ser at amino acid 60 was found in patients from families B-1 and B-2 (see Fig. $2 b$ ) and the two North Carolina patients, where the defect was designated as Factor IX $\mathrm{X}_{\text {Durham }}$ (9). The presence of a different polymorphism (Ala-148 vs. Thr) in the patients in family B-2 strongly argues for theirs, at least, being due to an independent mutation. It is otherwise possible that mutations leading to mild bleeding tendencies receive little selective pressure from the environment and thus persist and accumulate in the population.

In comparing sequences in the first growth factor-like region, residue 61 is Ile in the mouse instead of Ser in the human. The only other different amino acids are at residues 67, 74, 75, and 80 (26). The absence of a Pro-74 in murine Factor IX should lead to a significant conformational difference in the third disulfide loop-like structure; it is possible that the epitope involves both this site and bridges to partially include residues near or including Gly-60 in the first loop. The function of this region in Factor IX is unknown but its threedimensional structure has been approximated through an assumed homology of disulfide bond placement with epidermal growth factor as described by Cooke et al. (27) and Montelione et al. (28). Residues homologous to Factor IXs 60 and 74 are near each other at the two ends of an anti-parallel, beta-pleated sheet. On the other hand, long-range perturbations in structure may occur from single substitutions, such that residues outside of an epitope may affect the conformation of that epitope, as shown in cytochrome $c(29)$. A long range effect on conformation and surface charge density of 2D5's epitope might more readily account for the inverse degree of binding as related to ionic strength noted, since the addition of a simple hydroxylated methyl group would not lead to a major charge change at residue 60 . In either event, the Gly to Ser substitution is associated with clinically mild hemophilia with somewhat lower clotting activities than mildly reduced total antigen levels in four families. Furthermore, patient B-3 (Table I), for which DNA was not available, may well have the same point mutation. The only other defect known in exon IV is an Asp-47 to Gly in Factor IX Alabama (30). Immunoassays with 2D5 were normal in plasma from this patient (provided by Dr. Reisner).

Residue 248 is within the second and longest variable region of the heavy chain of Factor IXa (21). The epitope for the monoclonal anti-Factor IX, C10D (11), appears to involve more than the linear sequence around this residue as small synthetic peptides consisting of sequences through this region have not inhibited binding of Factor IX (Thompson, A. R., unpublished results). Nevertheless, the change from an Arg to Gln in two unrelated individuals makes this transition the likely cause of their moderately severe hemophilia B; this defect is designated Factor IX $\mathrm{X}_{\text {Seatle }}$. Since the levels of in vitro clotting activity and plasma antigen are essentially the same (in both individuals C-1 and C-2), it is possible that this defect results in an unstable Factor IX that is degraded rapidly, either before or after secretion. This might be similar to (though less severe than) an unstable hemoglobin that leads to one form of beta-thalassemia (31). The only other defect known within this region of Factor IX is a new stop codon-252 (32) which leads to severe hemophilia, as would be predicted from the inability to transcribe the balance of the active site including the active center Ser-365.

Of interest from these studies, two of the three mutations (B and C) were at $\mathrm{CpG}$ dinucleotides which are considered to be mutation hot spots in mammals (33). The mechanism of this phenomenon is speculated to involve methylation followed by deamination of the cytosine residue resulting in the formation of thymine. This type of mutation leads to a $G$ to $A$ transition in the complimentary strand (in these cases, the sense strand) and is found in nearly half of the reported point mutations in the Factor IX genes of patients with hemophilia B. 
The strategy of using selective amplification and DNA sequencing based on immunochemical sub-localization has been demonstrated as an effective method to identify gene mutations in these patients with hemophilia B. The possibility exists that the defects identified, particularly in patients $B$ and $\mathrm{C}$, might be incidental to the hemophilia, as the functional importance of the residues involved has not been demonstrated. The lack of altered reactivity in the immunoassays of Factor IX from over 100 normal males and other hemophilic patients, however, excludes all but rare polymorphisms as an alternative explanation for the findings. Patient $\mathrm{A}$ had a defect in what is predicted to be a critical Gla residue (24). Furthermore, defects in $B$ and $C$ were present in more than one family (in the case of B-1 vs. B-2 in families with a different Factor IX polymorphism), further reducing the probability that the defect is unrelated to hemophilia from about 1 in 100 to 1 in 10,000 or less. Finally, experience locally and in the literature has not shown these single-based transitions in several hemophilic or normal genes that have been cloned and sequenced for all coding and splice junction regions.

A similar strategy should apply to other genetic defects, such as milder forms of hemophilia A. There the gene is much larger and the epitopes for several MAbs have been localized (e.g., reference 34). It would first be necessary to screen affected individuals' plasmas with specific immunoassays.

\section{Acknowledgments}

The authors thank Dr. Reisner and Dr. Smith for their MAb preparations and Drs. Brownlee, Furie, and Reisner for patient samples. The technical assistance of Ms. Sunday Stray and Ms. Ann Weinmann is gratefully appreciated.

This study was supported by National Institutes of Health grant HL-31193 and American Heart Association grant 88-0805 (to Dr. Thompson), and the March of Dimes National Foundation grant 6-463 (to Dr. Chen and Dr. Scott).

\section{References}

1. Thompson, A. R. 1977. Factor IX antigen by radioimmunoassay. Abnormal Factor IX protein in patients on warfarin therapy and with hemophilia B. J. Clin. Invest. 59:900-910.

2. Brownlee, G. G. 1988. Haemophilia B: a review of patient defects, diagnosis with gene probes and prospects for gene therapy. $R e$ cent Adv. Haematol. 5:251-264.

3. Haldane, J. B. S. 1947. The mutation rate of the gene for hemophilia and its segregation ratios in males and females. Ann. Eugen. 13:262-271.

4. Bentley, A. K., D. J. G. Rees, C. Rizza, and G. G. Brownlee. 1985. Defective propeptide processing of blood clotting Factor IX caused by mutation of arginine to glutamine at position -4. Cell. 45:343-348.

5. Ware, J., H. Liebman, C. Kasper, J. Graham, B. C. Furie, B. Furie, and D. W. Stafford. 1986. Genetic characterization of a hemo-

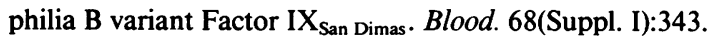

6. Liddell, M. B., D. P. Lillicrap, I. R. Peake, and A. L. Bloom. 1988. Factor IX Troed-Y-Rhiw, a functionless high molecular weight variant, is caused by a mutation in the pro-sequence at position -4. Br. J. Haematol. 69:150. (Abstr.)

7. Spitzer, S. G., U. R. Pendurthi, C. K. Kasper, and S. P. Bajaj.

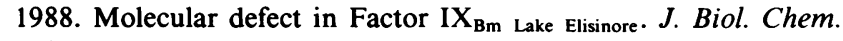
263:10545-10548.

8. Ware, J., L. Davis, D. Frazier, S. P. Bajaj, and D. W. Stafford. 1988. Genetic defect responsible for the dysfunctional protein. Factor IX Long Beach. Blood. 72:820-822.
9. Denton, P. H., D. M. Fowlkes, S. T. Lord, and H. M. Reisner. 1988. Hemophilia B Durham. A mutation in the first EGF-like domain of Factor IX that is characterized by polymerase chain reaction. Blood. 72:1407-1411.

10. Smith, K. J., A. R. Thompson, B. A. McMullen, D. Frazier, S. W. Lin, D. W. Stafford, W. Kisiel, S. N. Thibodeau, S.-H. Chen, and L. F. Smith. 1987. Carrier testing in hemophilia B with an immunoassay which distinguishes a prevalent Factor IX dimorphism. Blood. 70:1006-1013.

11. Thompson, A. R. 1983. Monoclonal antibody to an epitope on the heavy chain of Factor IX missing in three hemophilia B patients. Blood. 62:1027-1034.

12. Frazier, D., S. W. Lin, J. Ware, K. Smith, H. Reisner, M. DeSerres, A. Wallmark, R. Ljung, I. M. Nilsson, and D. W. Stafford. 1987. Mapping of 6 monoclonal antibodies to human Factor IX. Thromb. Haemostasis. 58:207.

13. Smith, K. J., and K. Ono. 1984. Monoclonal antibodies to Factor IX: characterization and use in immunoassays for Factor IX. Thromb. Res. 33:211-224.

14. Geddes, V. A., B. F. Le Bonniec, G. V. Louie, G. D. Brayer, A. R. Thompson, and R. T. A. MacGillivray. 1989. A moderate form of hemophilia $B$ is caused by a novel mutation in the protease domain of Factor IX Vancouver. J. Biol. Chem. 264:4689-4697.

15. Bray, G. L., A. F. Weinmann, and A. R. Thompson. 1986. Calcium specific immunoassays for Factor IX: reduced levels of antigen in patients with vitamin K disorders. J. Lab. Clin. Med. 107:269278.

16. Chen, S.-H., S. Yoshitake, P. F. Chance, G. L. Bray, A. R. Thompson, C. R. Scott, and K. Kurachi. 1985. An intragenic deletion of the Factor IX gene in a family with hemophilia B. J. Clin. Invest. 76:2161-2164.

17. Saiki, R. K., D. H. Gelfand, S. Stoffel, S. J. Scharf, R. G. Higuchi, T. T. Horn, K. B. Mullis, and H. A. Erlich. 1988. Primer-directed enzymatic amplification of DNA with a thermostable DNA polymerase. Science (Wash. DC). 239:487-491.

18. Sanger, F., S. Nicklen, and A. R. Coulson. 1977. DNA sequencing with chain-terminating inhibitors. Proc. Natl. Acad. Sci. USA. 74:5463-5467.

19. Smith, K. J., C. Singaraju, and L. F. Smith. 1987. Factor IX metal ion-dependent antigen assays for measurement of warfarin effect. Am. J. Clin. Pathol. 87:370-376.

20. Thompson, A. R., H. M. Reisner, D. W. Stafford, M. Vidaud, and M. Goossens. 1988. Binding of hemophilic Factor IX's to a monoclonal anti-IX helps localize the epitope and characterize defects. Clin. Res. 36:570A.

21. Furie, B., D. H. Bing, R. J. Feldmann, D. J. Robison, J. P. Burnier, and B. C. Furie. 1982. Computer-generated models of blood coagulation Factor $\mathrm{Xa}$, Factor IXa, and thrombin based upon structural homology with other serine proteases. J. Biol. Chem. 257:38753882.

22. Yoshitake, S., B. G. Schach, D. C. Foster, E. W. Davie, and K. Kurachi. 1985. Nucleotide sequence of the gene for human Factor IX (antihemophilic factor B). Biochemistry. 24:3736-3750.

23. Koeberl, D. D., J.-M. Buerstedde, E. S. Stoflet, C. D. K. Bottema, and S. S. Sommer. 1988. Direct sequence analysis of the Factor IX gene in multiple hemophiliacs and unaffected individuals by genomic amplification with transcript sequencing (GAWTS). Am. J. Hum. Genet. 43:A91.

24. Borowski, M., B. C. Furie, and B. Furie. 1986. Distribution of $\gamma$-carboxyglutamic acid residues in partially carboxylated human prothrombins. J. Biol. Chem. 261:1624-1628.

25. Duiguid, D. L., M. J. Rabiet, B. C. Furie, H. A. Liebman, and B. Furie. 1986. Molecular basis of hemophilia B: a defective enzyme due to an unprocessed propeptide is caused by a point mutation in the Factor IX precursor. Proc. Natl. Acad. Sci. USA. 83:5803-5807.

26. Wu, S. M., J. Ware, and D. W. Stafford. 1988. Sequence of mouse Factor IX cDNA. Blood. 72(Suppl. I):314a.

27. Cooke, R. M., A. J. Wilkinson, M. Baron, A. Pastore, M. J. 
Tappin, I. D. Campbell, H. Gregory, and B. Sheard. 1987. The solution structure of human epidermal growth factor. Nature (Lond.). 327:339-341.

28. Montelione, G. T., K. Wuthrich, E. C. Nice, A. W. Burgess, and H. A. Scheraga. 1987. Solution structure of murine epidermal growth factor: determination of the polypeptide backbone chain-fold by nuclear magnetic resonance and distance geometry. Proc. Natl. Acad. Sci. USA. 84:5226-5230.

29. Collawn, J. F., C. J. A. Wallace, A. E. I. Proudfoot, and Y. Paterson. 1988. Monoclonal antibodies as probes of conformational changes in protein-engineered cytochrome $c$. J. Biol. Chem. 263:8625-8634.

30. Davis, L. M., R. A. McGraw, J. L. Ware, H. R. Roberts, and D. W. Stafford. 1987. Factor IX $\mathrm{X}_{\text {Alabama }}$. A point mutation in a clotting protein results in hemophilia B. Blood. 69:140-143.
31. Adams, J. G., M. H. Steinberg, L. A. Boxer, R. L. Baehner, B. G. Forget, and G. A. Tsistrakis. 1979. The structure of hemoglobin Indianapolis [ $\beta 112(\mathrm{G} 14)$ arginine] J. Biol. Chem. 254:3479-3482.

32. Chen, S.-H., C. R. Scott, E. Lovrien, and K. Kurachi. 1989. Factor IX-Portland: a nonsense mutation (CGA $\rightarrow$ TGA) resulting in hemophilia B. Am. J. Hum. Genet. 44:567-569.

33. Youssoufian, H., H. H. Kazazian, D. G. Phillips, S. Aronis, G. Tsftis, V. A. Brown, and S. E. Antonarakis. 1986. Recurrent mutations in haemophilia A give evidence for CpG mutation hotspots. Nature (Lond.). 324:380-382.

34. Scandella, D., S. DeGraaf-Mahoney, M. Mattingly, D. Roeder, L. Timmons, and C. A. Fulcher. 1988. Epitope mapping of human factor VIII inhibitor antibodies by deletion analysis of factor VIII fragments expressed in Escherichia coli. Proc. Natl. Acad. Sci. USA. 85:6152-6156. 